\title{
Comparison of Local Anesthetic-Cyclodextrin Non-Covalent Complexes Using Capillary Electrophoresis and Electrospray Ionization Mass Spectrometry
}

\author{
Yazen Al-nouti and Michael G. Bartlett \\ Department of Pharmaceutical and Biomedical Sciences, College of Pharmacy, The University of Georgia, \\ Athens, Georgia, USA
}

\begin{abstract}
Non-covalent complexes between three derivitized cyclodextrins (CD's) and six local anesthetics were studied using capillary electrophoresis (CE) and electrospray ionization mass spectrometry (ESI-MS). The CE study was performed using the complete filling technique (CFT). A comparison between the migration data from CE and ESI-MS inclusion complex peak abundances was made representing the association between local anesthetics and CD's in the solution and the gas phase, respectively. The results from this study showed comparable behavior of the complexes in the $\mathrm{CE}$ and mass spectrometer, indicating similarity in the parameters controlling the stability of these complexes. Therefore, the formation of specific non-covalent complexes, as shown in this study, could be used to predict the behavior of a complexing agent with a substrate in the solution phase by observing data obtained from ESI-MS. (J Am Soc Mass Spectrom 2002, 13, 928-935) (c) 2002 American Society for Mass Spectrometry
\end{abstract}

$\mathrm{T}$ The formation of specific non-covalent complexes by association of guest compounds within the cavity of host molecules is currently an area of great interest in the field of mass spectrometry. Most cyclodextrins (CD's) are made up of six, seven, or eight glucose monomers bound together by a 1,4 ether linkage. Cyclodextrins are gaining importance in asymmetric organic synthesis for their catalytic properties and in the pharmaceutical, cosmetic, agriculture, and food industries where their ability to improve the hydrophilicity of lipophilic compounds is of great importance [1]. Understanding the formation of these non-covalent complexes with CD's would assist in improving our understanding of this important interaction. Capillary electrophoresis (CE) and electrospray ionization mass spectrometry (ESI-MS) are two techniques that can be used together to give a comprehensive picture of the formation of these complexes.

Mass spectrometry has been a powerful tool in the study of non-covalent interactions. Electrospray ionization is a soft ionization technique, which therefore allows for the transfer of non-covalent complexes from the solution phase into the gas phase. The gas phase environment of the mass spectrometer has the advantage of avoiding

Published online June 13, 2002

Address reprint requests to Dr. M. G. Bartlett, Department of Pharmaceutical and Biomedical Sciences, College of Pharmacy, The University of Georgia, Athens, GA 30602-2352, USA. E-mail: bartlett@rx.uga.edu many interferences and allows for facile identification and detection of host-guest complexes [2]. Numerous studies have reported the observation of intact non-covalent complexes and this information was used to provide insight into specific non-covalent associations in solution [1-6]. However, the production of non-specific adducts have been reported too, leading to concerns over the ability of mass spectrometry to be used to study non-covalent associations $[7,8]$.

If the ESI-MS is able to retain specific inclusion complexes in the same form as in the solution state, it could be a very effective tool to study the formation of these complexes $[1,8,9]$. Since ESI-MS can be applied to solution conditions close to those of physiological interest, it can provide information on enzyme-substrate, receptor-ligand and antigen-antibody interactions, as well as host-guest complexes [2, 10].

The correlation between the behavior of solution and gas phase complexes has been studied extensively over the past decade. Several approaches have been proposed to determine if the gas phase complex in question is a non-specific adduct or representative of a specific non-covalent interaction. The first approach was developed by Vouros and co-workers where substrates that lack the structural features favoring association with a complexing agent are compared to substrates that favor the formation of such complexes. In this case, the detection of any complexes would indicate the formation of electrostatic adducts during the electrospray 
process [7]. These weakly bound complexes would also decompose easily using low energy collision induced dissociation studies (CID) [11]. Second, hydrogen/deuterium $(\mathrm{H} / \mathrm{D})$ exchange has been used by comparing the average number of exchanges for the individual units comprising the complex with the average number of exchanges for the complex itself. If a complex is formed during the ionization process, the average number of $\mathrm{H} / \mathrm{D}$ atoms exchanged in a complex should equal the sum of $H / D$ atoms exchanged in each of the components [12]. Third, an on-line separation technique like gel permeation was applied to remove the free guest and the free complexing agent from the specific complex in solution. This technique guarantees that neither the guest nor the host is found free in the solution to form non-specific adducts during ionization [13]. The fourth approach involves masking or unfolding an active site that is known to bind specifically to the complexing agent. If the detected complex still exists after the deconformation of the active site, it indicates formation of non-specific aggregates [10].

A previous study performed in this lab showed the formation of specific non-covalent complexes of a group of acidic hypnotic agents [3]. In this study, we extend this approach to a group of basic local anesthetics in an attempt to determine whether the behavior previously observed was limited to the acidic compounds or is more universal in its applicability.

The six local anesthetics under study have structural features that favor binding with the $C D$ cavity in ammonium formate buffer. Ammonium formate was chosen because of earlier reports that it suppresses the formation of non-specific aggregates in positive ion electrospray [13]. To show the correlation between the behavior of specific non-covalent complexes in the liquid and the gas phase, this study is divided into two parts. First, the comparison of the order of association constants of the different analytes with each $C D$ as measured by $\mathrm{CE}$, with the order of their peak intensity ratios as measured by ESI-MS. Second, comparing the relative affinities of each analyte to three different complexing agents (CD's) as calculated by $\mathrm{CE}$ and ESI-MS. If the behavior of the solution phase and the gas phase complexes are similar, there is evidence for formation of specific non-covalent inclusions.

\section{Experimental}

\section{Materials and Reagents}

Proparacaine, meprilcaine, lidocaine, prilocaine, dibucaine, dyclonine, heptakis(2,3,6-tri-o-methyl)- $\beta$-cyclodextrin, heptakis(2,6-di-o-methyl)- $\beta$-cyclodextrin, and ammonium formate $97 \%$ were obtained from Aldrich Chemical Company, Inc. (Milwaukee, WI). Formic acid 90.3\% was obtained from J. T. Baker (Phillipsburg, NJ). Nylon membrane syringe filters $(0.2 \mu \mathrm{m})$ were purchased from Fisher Scientific (Pittsburgh, PA). Methyl$\beta$-cyclodextrin ( $\mathrm{M} \beta \mathrm{CD})$, hydroxypropyl- $\beta$-cyclodextrin, and $\gamma$-cyclodextrin were kindly supplied by Cerastar USA, Inc. (Indianapolis, IN)

\section{Mass Spectrometry}

A Quattro II (Micromass, Inc., Beverly, MA) triple quadrupole mass spectrometer equipped with the Z-spray electrospray ionization source was used for all the mass spectrometry experiments. The mass spectrometer was set to the positive ion mode of operation, with the source temperature at $150^{\circ} \mathrm{C}$. The cone and the ion source capillary voltages were adjusted to maximize the signal/noise ratio of the analyte-CD complex, which were found to be $20 \mathrm{~V}$ and $3000 \mathrm{~V}$, respectively. The data were collected in the multi-channel analysis (MCA) mode and represent the summation of ten individual mass spectral scans. Direct flow injection was carried out using a syringe infusion pump (KD Scientific, Boston, MA) to deliver sample at a flow rate of $15 \mu \mathrm{L} / \mathrm{min}$ into the ESI source.

\section{Capillary Electrophoresis}

A P/ACE system 5000 CE (Beckman, Inc., Fullerton, $\mathrm{CA}$ ) equipped with an UV detector was used for all CE experiments. The separation capillary was uncoated fused-silica tubing (Polymicro Technologies, Phoenix, AZ) of $75 \mu \mathrm{m}$ i.d.. The total length of the capillary was $57 \mathrm{~cm}$ with a $50 \mathrm{~cm}$ effective length, which represents the distance from the front of the capillary to the detector. The capillary was thermostated at $25^{\circ} \mathrm{C}$ and detection was carried out using the UV detector at 254 $\mathrm{nm}$. Samples were introduced into the capillary by pressure, $0.5 \mathrm{psi}(3.4 \mathrm{kPa})$, for $5 \mathrm{~s}$. Separation was found to be optimum at $15 \mathrm{kV}$ toward the cathodic electrode. Electrolyte backgrounds (EBG) over the cyclodextrin concentration range from $14-60 \mathrm{mM}$ were prepared in $100 \mathrm{mM}$ ammonium formate buffer $\mathrm{pH} 2$.

\section{Preparation of Stock and Standard Solutions}

For the CE experiments the buffer system was prepared as $100 \mathrm{mM}$ ammonium formate in water, which was then adjusted to $\mathrm{pH} 2$ using concentrated formic acid. The analytes were dissolved in 10-fold diluted ammonium formate buffer $(10 \mathrm{mM})$ to prepare $1 \mathrm{mg} / \mathrm{ml}$ stock solutions. Fifty $\mu \mathrm{g} / \mathrm{ml}$ samples concentrations were then prepared from each respective stock to be injected into the CE. Dissolving the analyte in a solvent of lower conductivity (lower concentration) will cause them to be stacked in a narrower zone, producing sharper peaks and allowing for lower limits of detection. Cyclodextrin (CD) stock solutions were prepared in the $100 \mathrm{mM}$ ammonium formate to give a final concentration range from $14-60 \mathrm{mM}$. All solutions were filtered prior to use with a $0.2 \mu \mathrm{m}$ Nylon filter syringe.

For the MS experiments, $20 \mathrm{mg} / \mathrm{ml}$ cyclodextrin stock solutions were prepared in $100 \mathrm{mM}$ ammonium formate buffer. Analyte stock solutions were prepared 


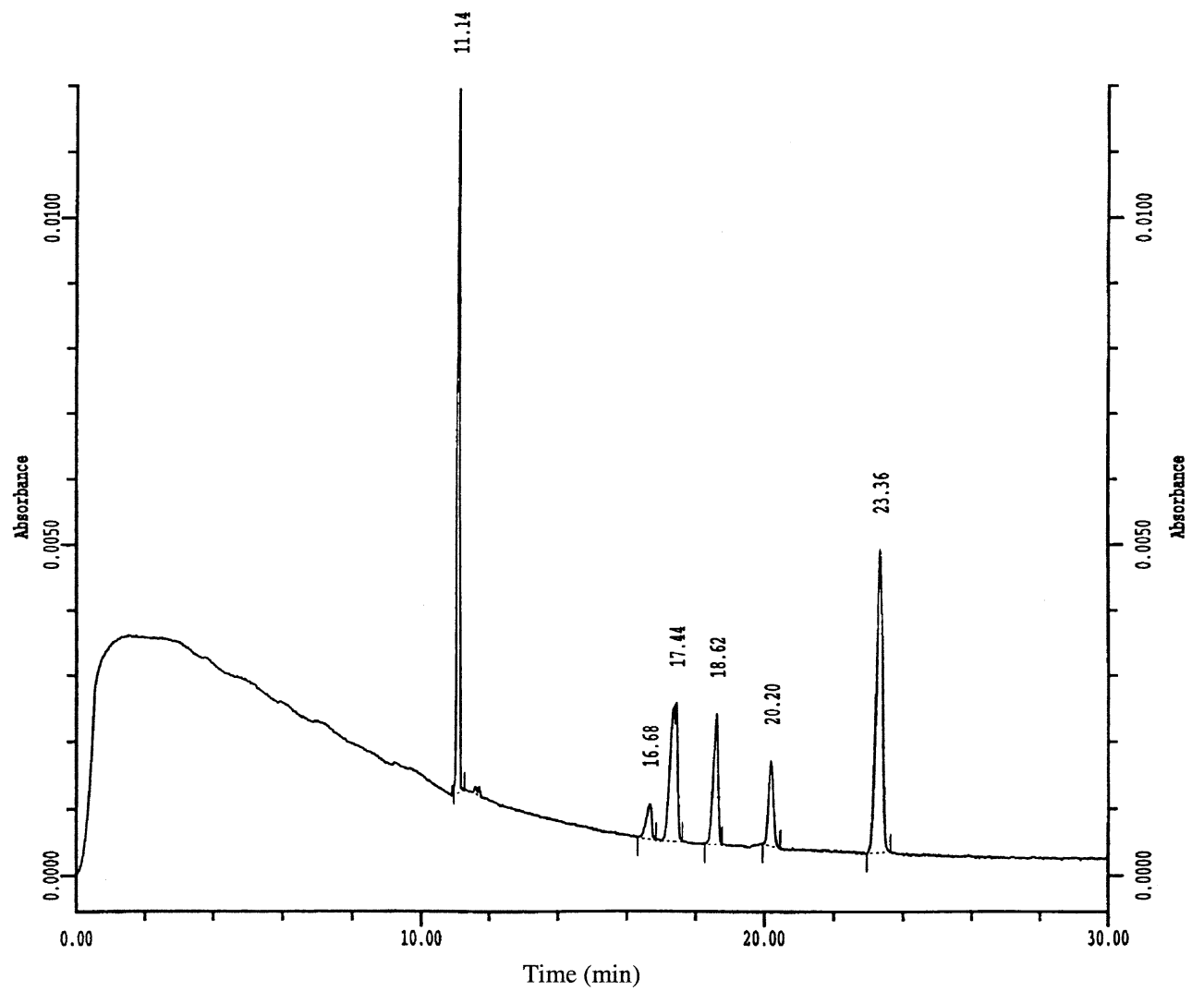

Figure 1. Typical electropherogram showing the resolution of the six local anesthetics, proparacaine (11.14), lidocaine (16.68), prilocaine (17.44), meprilcaine (18.62), lidocaine (20.20), and dyclonine (23.36), on a $50 \mathrm{~cm}, 75 \mu \mathrm{m}$ uncoated silica capillary. The buffer contained $33.3 \mathrm{mM} \mathrm{M} \beta C D$ in $100 \mathrm{mM}$ ammonium formate buffer $(\mathrm{pH} 2)$ with detection at $254 \mathrm{~nm}$. The separation voltage was $15 \mathrm{kV}$.

in the same way as for the CE. Appropriate volumes of the $\mathrm{CD}$ stock and the analyte stock solution were pipetted to give 1:100 $\mathrm{w} / \mathrm{w}$ ratio of the analyte to the CD.

\section{Electrophoretic Conditions}

Initially an uncoated fused silica capillary was rinsed with $1 \mathrm{M} \mathrm{HCl}$ for $2 \mathrm{~h}$, with water for $3 \mathrm{~h}$, with $0.1 \mathrm{M}$ $\mathrm{NaOH}$ for $30 \mathrm{~min}$, and finally with water for $30 \mathrm{~min}$. The capillary was flushed with water, running buffer, and with water again, 2 min each before the buffered CD solution was introduced into the capillary. The buffer system was $100 \mathrm{mM}$ ammonium formate ( $\mathrm{pH} 2)$. The buffer in the outlet and inlet vials was replaced after each run to avoid ion depletion allowing better reproducibility of the analytes migration times. The ammonium formate buffer was prepared fresh every morning and the capillary was stored dry overnight.

The principle of analyte stacking was applied to this study by dissolving the analyte in a solvent of lower conductivity than the run buffer. The electric current increases by decreasing the solvent conductivity, which causes the analyte to move faster in the sample plug until encountering the boundary of the higher concentration buffer $[3,14]$. This approach causes the analyte to be stacked in a narrow zone producing sharper peaks. Figure 1 shows a representative electropherogram.

\section{The Complete Filling Technique (CFT)}

For all CE experiments in this study the complete filling technique (CFT) was used. The CFT was developed as a modification of the partial filling technique (PFT). The PTF was introduced by Valtcheva et al. in 1993 [15], modified by Tanaka and Terabe, in 1995 [16], and applied by Amini et al. in 1997 [17] and again in 1999 [18]. In the PFT, the capillary is filled with the selector solution up to the detection window, the sample injection is then applied, and finally the separation process is pursued by applying the voltage while the capillary ends are dipped into the electrolyte background (EBG) containing no CD selector [14,17-20]. The PFT was originally developed to avoid detection interferences by a UV active selector by choosing separation conditions that held the selector stationary or moving away from the detection window $[19,20]$. Since the selector used in this study (cyclodextrin) has no UV interference or low UV absorbtivity, the capillary can be entirely filled with the CD solution prior to sample introduction. The separation was performed in the plain EBG [18]. The use of the CFT significantly decreases the consumption of the cyclodextrin selector used (this whole study was conducted using milligrams of CD), which is of great importance when expensive selectors are used.

When using the PFT, a separation zone of a specific 


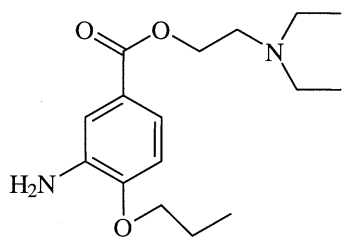

Proparacaine $(\mathrm{pKa}=9.1)$

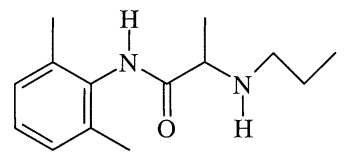

Prilocaine $(\mathrm{pKa}=7.9)$

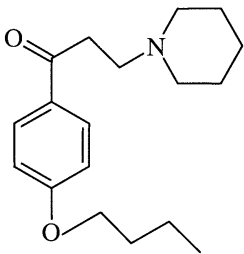

Dyclonine $(\mathrm{pKa}=8.2)$

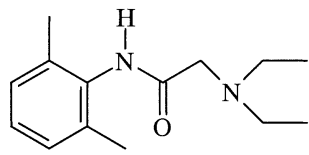

Liocaine $(\mathrm{pKa}=7.8)$

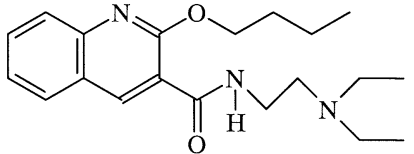

Dibucaine $(\mathrm{pKa}=8.8)$

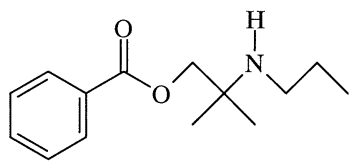

Meprilcaine $(\mathrm{pKa}=7.8)$

Figure 2. Chemical structures of the local anesthetics.

length is used by applying the $\mathrm{CD}$ selector solution with a low pressure for a specific amount of time. This method is usually very time consuming [18]. We chose to use CFT rather than PFT, since (1) previous studies indicate that the longer the separation zone the better the resolution [18, 19], and (2) the CD selector is UV-inert. The capillary was flushed with the CD solution using high pressure for $2 \mathrm{~min}$, which guarantees complete filling of the whole capillary length. The voltage was applied after the sample was introduced into the capillary, and the capillary ends are dipped in the input and the output vials filled with the EBG containing no $\mathrm{CD}$ selector.

\section{Results and Discussion}

Six local anesthetics have been studied; their structures and $\mathrm{pKa}$ values are shown in Figure 2. Ammonium acetate, monobasic sodium phosphate and ammonium formate buffer systems were investigated to separate the local anesthetics. Figure 1 illustrates a representative electropherogram where the analytes were separated in formate buffer containing $33.3 \mathrm{mM} \mathrm{M} \beta \mathrm{CD}$. Ammonium formate at $\mathrm{pH} 2$ was found to provide the sharpest peaks with the best chiral resolution of the single chiral analyte, prilocaine. Acidic $\mathrm{pH}$ was found to provide improved CE separations because it was able to ionize the basic analytes. It was noticed that $\mathrm{CE}$ resolution increased with decreasing $\mathrm{pH}$. Some of the peaks were partially overlapped and base line resolution of all the peaks was not achieved until we went down to $\mathrm{pH}$ 2. At $\mathrm{pH} \mathrm{2,} \mathrm{the} \mathrm{electroosmotic} \mathrm{flow} \mathrm{(EOF)} \mathrm{is} \mathrm{almost}$ totally suppressed, the analytes are all positively charged and the neutral CD's are almost immobile. The uncomplexed positively charged analyte moves toward the cathodic end (detector end) by its own native electrophoretic velocity. The greater the interaction with the $\mathrm{CD}$ the longer the migration time of the analyte [21].

$\mathrm{M} \beta \mathrm{CD}$, heptakis-di-o-methyl, and tri-o-methyl- $\beta$-CD were studied over the concentration range of $10-80$ $\mathrm{mM}$. The relationship between the migration time and the $\mathrm{CD}$ concentration was linear over the range from 15-65 mM. All three CD's were able to separate the six analytes. However, base line resolution of the enantiomers of prilocaine was achieved when using heptakis di-o-methyl- $\beta-C D$ and $M \beta C D$ at concentrations greater than $50 \mathrm{mM}$.

\section{Calculation of the Local Anesthetic-CD Interaction by CE and ESI-MS}

To determine the extent of complexation or association between the CD selector and the analyte (local anesthetic) in the solution phase, the association constant (K) was determined. $\mathrm{K}$ values were calculated using the general equation of micellar electrokinetic capillary chromatography (MECC). This equation expresses the relationship between the apparent velocity of the complex and the apparent velocity of the free analyte and the free $C D$ [22]:

$$
\mathrm{V}_{\mathrm{obs}}=\mathrm{F}_{\mathrm{aq}}\left(\mathrm{V}_{\text {app.A }}\right)+\mathrm{F}_{\mathrm{mc}}\left(\mathrm{V}_{\text {app.mc }}\right)
$$

Where $V_{o b}$ is equal to the observed complex velocity, $\mathrm{V}_{\text {app·A }}$ is equal to the apparent drug velocity in the $C D$ free buffer, $V_{\text {app-mc }}$ is equal to the apparent $C D$ velocity in the drug free buffer and $\mathrm{F}_{\mathrm{aq}}, \mathrm{F}_{\mathrm{mc}}$ are equal to the molar fraction factor of the free drug in solution $\left(\mathrm{n}_{\mathrm{aq}} /\right.$ $\left.\mathrm{n}_{\text {total }}\right)$ and drug complexed with the CD $\left(\mathrm{n}_{\mathrm{mc}} / \mathrm{n}_{\text {total }}\right)$ respectively. The association constant $(\mathrm{K})$ can be determined using the following eq [22]:

$$
\mathrm{KC}=\mathrm{n}_{\mathrm{mc}} / \mathrm{n}_{\mathrm{aq}}
$$

Where $C$ is the CD concentration and $n_{m c} / n_{a q}$ is the molar fraction of the complexed drug to the uncomplexed drug. The following equation expresses the relationship between the net analyte velocity $\left(\mathrm{V}_{\text {app }}\right)$, the electrophoretic velocity $\left(\mathrm{V}_{\mathrm{ep}}\right)$, and the electroosmotic velocity $\left(\mathrm{V}_{\mathrm{eo}}\right)$ [22]: 
Table 1. Association constant values $(\mathrm{K})$ as calculated from $\mathrm{CE}$ and normalized complexs intensities values (n. $\mathrm{R}_{\mathrm{ms}}$ ) averages

\begin{tabular}{|c|c|c|c|c|c|c|}
\hline & \multicolumn{2}{|c|}{$\mathrm{M} \beta \mathrm{CD}$} & \multicolumn{2}{|c|}{ Heptakis-di-o-methyl- $\beta$-CD } & \multicolumn{2}{|c|}{ Heptakis-tri-o-methyl- $\beta$-CD } \\
\hline & CE (K) & ESI-MS (n. $\left.R_{m s}\right)$ & CE & ESI-MS ( $n \cdot R_{m s}$ & CE & ESI-MS (n. $\left.R_{m s}\right)$ \\
\hline Proparacaine & 1.55 & 0.549 & 0.784 & 0.513 & 0.738 & 0.215 \\
\hline Lidocaine & 1.82 & 0.743 & 1.16 & 0.591 & 0.779 & 0.310 \\
\hline Prilocaine & 1.95 & 0.849 & 1.98 & 0.677 & 0.784 & 0.397 \\
\hline Meprilcaine & 2.28 & 0.915 & 2.83 & 0.752 & 0.816 & 0.541 \\
\hline Dibucaine & 4.23 & 1 & 4.20 & 1 & 1.50 & 1 \\
\hline Dyclonine & 4.26 & 2.450 & 4.23 & 1.401 & 1.88 & 4.430 \\
\hline
\end{tabular}

Note: All complex intensities values were normalized to the responses from dibucaine.

$$
\mathrm{V}_{\mathrm{app}}=\mathrm{V}_{\mathrm{ep}}+\mathrm{V}_{\mathrm{eo}}
$$

By substituting eq 2 and eq 3 into eq 1, we are able to express the velocity of the analyte-CD complex in terms of the association constant, CD concentration, and electrophphoretic velocity of uncomplexed species [22]:

$$
\begin{aligned}
\mathrm{V}_{\mathrm{ob}}= & (1 / 1+\mathrm{KC})\left(\mathrm{V}_{\text {ep.a }}+\mathrm{V}_{\mathrm{eo}}\right)+(\mathrm{KC} / \mathrm{KC}+1) \\
& \cdot\left(\mathrm{V}_{\text {ep.mc }}+\mathrm{V}_{\text {eo }}\right)
\end{aligned}
$$

Since $\mathrm{pH} 2$ was used, which is low enough to suppress the electroosmotic flow by not allowing enough deprotonation or ionization of the silinol groups that form the internal surface of the capillary, the $\mathrm{V}_{\mathrm{eo}}$ term equals zero $[21,23,24]$. This condition was proven by using a neutral marker that was not observed by the UV detector following $5 \mathrm{~h}$ of analysis time. $\mathrm{V}_{\text {ep.mc }}$ also equals zero since all the CD's used are neutral. Applying these conditions to eq 4 , we can simplify it to eq 5 :

$$
\mathrm{V}_{\mathrm{ob}}=(1 / 1+\mathrm{KC})\left(\mathrm{V}_{\text {app.a }}\right)
$$

The relationship between the velocity and the migration time is [22]:

$$
\mathrm{V}=\mathrm{L} / \mathrm{T}
$$

Where $\mathrm{L}$ is the total capillary length and $\mathrm{T}$ is the apparent migration time. Substituting eq 6 into eq 5 yields an equation, which relates observed migration times to $\mathrm{CD}$ concentration:

$$
\mathrm{T}_{\mathrm{obs}}=\mathrm{T}_{\text {app.A } \mathrm{A}} \mathrm{KC}+\mathrm{T}_{\text {app.A }}
$$

Where $T_{o b}$ is equal to the migration time observed in the presence of the $C D, T_{a p p \cdot A}$ is equal to the observed migration time in the absence of the $\mathrm{CD}$.

By plotting the $T_{\text {obs }}$ on the $y$-axis versus $C$ in the $\mathrm{x}$-axis and by dividing the slope by $\mathrm{T}_{\text {app:A }}$ we were able to calculate the association constant values $(\mathrm{K})$ for all the local anesthetics with the CD's. The results are shown in Table 1. In ESI-MS the extent of complexation can be determined by calculating the $\mathrm{CD}$ fraction complexed with the analyte by measuring the ratio of $\mathrm{CD}$-analyte complex peak height to the sum of the complexed and the free $\mathrm{CD}\left(\mathrm{R}_{\mathrm{ms}}\right)[4,5]: \mathrm{R}_{\mathrm{ms}}=\mathrm{CD}-$ analyte complex peak height/CD-analyte complex peak height + free CD peak height. Higher $R_{m s}$ values indicate stronger analyte-CD association, therefore $R_{m}$ is a measure of the strength of the association.

\section{Mass Spectrometry Data}

$\mathrm{M} \beta \mathrm{CD}$ and heptakis di-methoxy- $\beta$-CD have a distribution of peaks in the mass spectrum separated by 14 mass units difference representing different degrees of substitution of the methyl groups. Each CD-analyte complex peak height was divided by the corresponding free $C D$ peak height and the average for each set was used to calculate $R_{m s}$. The standard conditions to compare complex intensities of different analytes would be to apply the same $C D$ with all the analytes in the same injection, thus creating a competitive environment for the different substrates to compete for binding to the $\mathrm{CD}$ each according to its own relative affinity. Unfortunately, lidocaine (235.5 Da) and meprylcaine (236.5 Da) have almost the same molecular weight, differing by one mass unit. Furthermore, prilocaine (221.5 Da) is 14 mass units lower than lidocaine which is the exact mass difference between the peaks corresponding to the degree of methylation of the $\mathrm{CD}$. It was therefore not possible to measure $R_{m s}$ values for the six analytes in

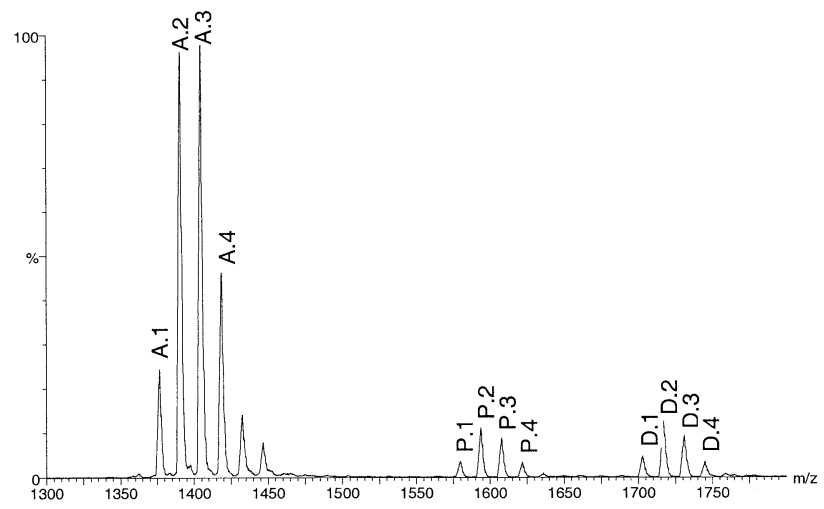

Figure 3. Mass spectrum of a mixture of prilocaine, dibucaine, and $\mathrm{M} \beta C \mathrm{C}$ in $100 \mathrm{mM}$ ammonium formate buffer ( $\mathrm{pH}$ 2). The peaks marked " $A$ " represent signals from free $M \beta C D$. The peaks marked " $\mathrm{P}$ " represent $\mathrm{CD}$-prilocaine complexes and the peaks marked " $\mathrm{D}$ " represent various $\mathrm{CD}$-dibucaine complexes. 
Table 2. Normalized complex intensities values (n. $R_{m s}$ ) of the analytes with $M \beta C D$ as calculated from the ESI-MS data

\begin{tabular}{|c|c|c|c|c|c|c|c|c|c|c|c|c|}
\hline \multirow{2}{*}{$\begin{array}{l}\mathrm{CD} \\
\underline{m / z}\end{array}$} & \multicolumn{2}{|c|}{ Proparacaine } & \multicolumn{2}{|c|}{ Lidocaine } & \multicolumn{2}{|c|}{ Prilocaine } & \multicolumn{2}{|c|}{ Meprilcaine } & \multicolumn{2}{|c|}{ Dibucaine } & Dyclonine & \multirow[b]{2}{*}{$\mathrm{n} \cdot \mathrm{R}_{\mathrm{ms}}$} \\
\hline & $m / z$ & $\mathrm{n} \cdot \mathrm{R}_{\mathrm{ms}}$ & $m / z$ & $\mathrm{n} \cdot \mathrm{R}_{\mathrm{ms}}$ & $m / z$ & $\mathrm{n} \cdot \mathrm{R}_{\mathrm{ms}}$ & $m / z$ & $\mathrm{n} \cdot \mathrm{R}_{\mathrm{ms}}$ & $m / z$ & $\mathrm{n} \cdot \mathrm{R}_{\mathrm{ms}}$ & $m / z$ & \\
\hline 1376 & 1653 & 0.810 & 1593 & 0.716 & 1579 & 0.782 & 1594 & 0.916 & 1702 & 1 & 1648 & - \\
\hline 1390 & 1667 & 0.383 & 1607 & 0.775 & 1593 & 0.843 & 1608 & 0.910 & 1716 & 1 & 1662 & 2.501 \\
\hline 1404 & 1681 & 0.558 & 1621 & 0.757 & 1607 & 0.885 & 1622 & 0.853 & 1730 & 1 & 1676 & 2.398 \\
\hline 1418 & 1695 & 0.444 & 1635 & 0.724 & 1621 & 0.886 & 1636 & 0.980 & 1744 & 1 & 1690 & - \\
\hline Average & & 0.549 & & 0.743 & & 0.849 & & 0.915 & & 1 & & 2.45 \\
\hline $\mathrm{RSD}^{\mathrm{a}}$ & & 31.77 & & 5.31 & & 7.69 & & 6.11 & & 1 & & 2.51 \\
\hline
\end{tabular}

Note: Not all data points were available due to the overlap of the signal. This is indicated by (-). All complex intensities values were normalized to the responses from dibucaine.

${ }^{a}$ To calculate RSD values, data from the three trials were considered.

the same injection using our quadrupole instrument, because of the overlap of the peaks in the mass spectra. In order to simulate a partial competitive environment as much as possible, five injections of each analyte with the $C D$ were run. A representative mass spectrum is shown in Figure 3. Each injection contained dibucaine, which has the highest molecular weight and therefore does not overlap with any other analyte peaks, and one other local anesthetic. By normalizing each drug complex peak intensity to the peak intensity of the dibucaine complex, it was possible to calculate relative $R_{m s}$ values normalized to the dibucaine peak intensity. Each individual injection was repeated three times and the average was considered for the data in Tables 2, 3, and 4. The $\mathrm{R}_{\mathrm{ms}}$ data have standard deviations of less than $11 \%$ indicating the high reproducibility of the ESI-MS data. Proparacaine, however, shows a high standard deviation for its $R_{m s}$ values which is most likely due to the fact that it has a relatively low affinity to the CD's. It is also interesting to note that the measured $R_{m s}$ values do not differ because of the number of methyl side chains in the CD'S.

The second part of the study was to compare the relative affinities of each analyte separately, between the three CD's used. The standard conditions would normally be to apply the analyte with the three CD's together in a single injection again creating a competitive environment between the analytes and the CD's. Since the mass distributions of $M \beta C D$ and heptakis-dimethoxy- $\beta-C D$ were overlapping, it was not possible to create such an environment. Again the alternative was to create a partially competitive environment where $(\mathrm{M} \beta \mathrm{CD}$ and heptakis-tri-methoxy- $\beta-\mathrm{CD}$ ) and (heptakistri and di-methoxy- $\beta$-CD) were run as separate injections with each analyte.

By normalizing the height of $\mathrm{M} \beta \mathrm{CD}$-drug complex peak to the heptakis-tri-methoxy- $\beta-\mathrm{CD}$ and doing the same to heptakis-di-methoxy- $\beta-C D$, it was possible to calculate a relative $R_{m s}$ for each CD normalized to heptakis-tri-methoxy- $\beta$-CD with the heptakis-tri-methoxy- $\beta$-CD normalized $\mathrm{K}$ equal to one. These normalized values are shown in Table 5 .

\section{Formation of Specific Local Anesthetic-CD Non- Covalent Complexes}

An interesting feature of the ESI-MS binding of the analytes with the cyclodextrins is the reproducible loss of water molecule. This water loss is presumed to arise from displacement of water from the CD cavity. This water loss was not observed in our earlier studies with the bindings of barbiturates with CD's. However, that study did not use the same CD's and was conducted at a much higher $\mathrm{pH}$. It is not known if this loss of water is involved in the solution state interactions between the local anesthetics and the CD's.

All the local anesthetics chosen have structural features that favor binding with the $\beta-C D$ cavity. Previously, the formation of true host-guest inclusion com-

Table 3. Normalized complex intensities values (n. $\left.R_{m s}\right)$ of the analytes with heptakis-di-o-methyl- $\beta$-CD as calculated from the ESI-MS data

\begin{tabular}{|c|c|c|c|c|c|c|c|c|c|c|c|c|}
\hline \multirow{2}{*}{$\begin{array}{l}\mathrm{CD} \\
\overline{m / z}\end{array}$} & \multicolumn{2}{|c|}{ Proparacaine } & \multicolumn{2}{|c|}{ Lidocaine } & \multicolumn{2}{|c|}{ Prilocaine } & \multicolumn{2}{|c|}{ Meprilcaine } & \multicolumn{2}{|c|}{ Dibucaine } & \multicolumn{2}{|c|}{ Dyclonine } \\
\hline & $m / z$ & $\mathrm{n} \cdot \mathrm{R}_{\mathrm{ms}}$ & $m / z$ & $\mathrm{n} \cdot \mathrm{R}_{\mathrm{ms}}$ & $m / z$ & $\mathrm{n} \cdot \mathrm{R}_{\mathrm{ms}}$ & $m / z$ & $\mathrm{n} \cdot \mathrm{R}_{\mathrm{ms}}$ & $m / z$ & $\mathrm{n} \cdot \mathrm{R}_{\mathrm{ms}}$ & $m / z$ & $\bar{n} \cdot R_{\mathrm{ms}}$ \\
\hline 1348 & 1625 & 0.475 & 1565 & 0.599 & 1551 & 0.611 & 1566 & 0.695 & 1674 & 1 & 1620 & - \\
\hline 1362 & 1639 & 0.551 & 1579 & 0.568 & 1565 & 0.622 & 1580 & 0.737 & 1688 & 1 & 1634 & 1.465 \\
\hline 1376 & 1653 & 0.409 & 1593 & 0.614 & 1579 & 0.758 & 1594 & 0.836 & 1702 & 1 & 1648 & 1.336 \\
\hline 1390 & 1667 & 0.620 & 1607 & 0.583 & 1593 & 0.715 & 1608 & 0.740 & 1716 & 1 & 1662 & - \\
\hline Average & & 0.513 & & 0.591 & & 0.677 & & 0.752 & & 1 & & 1.401 \\
\hline $\mathrm{RSD}^{\mathrm{a}}$ & & 16.10 & & 10.13 & & 9.67 & & 8.87 & & 1 & & 4.61 \\
\hline
\end{tabular}

Note: not all data points were available due to the overlap of the signal. This is indicated by (-). All complex intensities values were normalized to the responses from dibucaine.

aTo calculate RSD values, data from the three trials were considered. 
Table 4. Normalized complex intensities values (n. $\left.\boldsymbol{R}_{\mathrm{ms}}\right)$ of the analytes with heptakis-tri-o-methyl- $\beta$-CD $(\mathrm{m} / z=1446)$ as calculated from the ESI-MS data

\begin{tabular}{lcccccc}
\hline & Proparacaine & Lidocaine & Prilocaine & Meprilcaine & Dibucaine & Dyclonine \\
\hline \hline$m / z$ & 1723 & 1663 & 1649 & 1664 & 1772 & 1718 \\
$\mathrm{n} . \mathrm{R}_{\mathrm{ms}}$ & 0.215 & 0.310 & 0.397 & 0.541 & 4.430 \\
$\mathrm{RSD}^{\mathrm{a}}$ & 11.53 & 7.85 & 9.41 & 9.84 & 1 & 7.36 \\
\hline
\end{tabular}

Note: All complex intensities values were normalized to the responses from dibucaine.

a To calculate RSD values, data from the three trials were considered.

plexes with $\beta-\mathrm{CD}$ had been reported [3, 25]. As observed in this study, the presence of an additional heterocyclic moiety in the anesthetic structure enhances the binding with the $\beta$-CD. This pattern was observed from the relatively high $\mathrm{K}$ values for dibucaine and dyclonine, which contain pyridine and piperidine rings respectively. The correlation between the analyte-CD complex behavior in the solution and the gas phases was studied in two steps. First, the relative affinities of the six compounds were compared to each $\mathrm{CD}$, separately. The decremental order of the values of association constants as calculated from the CE were, dyclonine, dibucaine, meprilcaine, prilocaine, lidocaine, and proparacaine. The complexes intensities observed in the ESI-MS for the different analytes decreased in the same order as seen in the CE, as can be noticed from Table 1. The second part was to study the effect of different CD's, by comparing the relative affinities of each analyte to three different $\mathrm{CD}^{\prime} \mathrm{s}$. The general trend was that the analytes have the greatest affinity for $\mathrm{M} \beta \mathrm{CD}$ and the lowest for the heptakis-tri-methoxy- $\beta$-CD except for meprilcaine and prilocaine, which have lower affinities for $M \beta C D$ relative to heptakis di-methoxy- $\beta-C D$. This trend, including the different meprilcaine and prilocaine behavior, could be observed from both the CE data, as shown in Table 1, as well as the ESI-MS data, as shown in Table 5. A cyclodextrin that has stronger affinity to one analyte than to another, as expressed by having higher $\mathrm{K}$ values in the $\mathrm{CE}$ experiments still gave a more intense signal with that analyte in the ESI-MS. Furthermore, the ESI-MS data shows that the interaction of the analytes with the CD's was not significantly affected by the degree of substitutions on the CD's. This indicates that the association was due to interaction with the $C D$ cavity and that the number of the side

Table 5. Normalized complex intensities values (n. $\left.R_{m s}\right)$ of each analyte with the $3 \mathrm{CD}^{\prime} \mathrm{s}$

\begin{tabular}{lrcc}
\hline & $\mathrm{M} \beta \mathrm{CD}$ & $\begin{array}{c}\text { Heptakis-di-o- } \\
\text { methyl- } \beta-\mathrm{CD}\end{array}$ & $\begin{array}{c}\text { Heptakis-tri-o- } \\
\text { methyl- } \beta \text {-CD }\end{array}$ \\
\hline \hline Proparacaine & 12.23 & 8.12 & 1 \\
Lidocaine & 22.70 & 10.21 & 1 \\
Prilocaine & 4.23 & 4.71 & 1 \\
Meprilcaine & 7.95 & 8.10 & 1 \\
Dibucaine & 8.42 & 6.79 & 1 \\
Dyclonine & 2.73 & 2.41 & 1 \\
\hline
\end{tabular}

Note: All complex intensities values were normalized to the responses from heptakis-tri-o-methyl- $\beta$-CD. chains does not affect the access of the analyte to the $C D$ core. These correlations suggest that the complexes detected in the ESI-MS experiments were passed intact from the solution phase. Therefore these complexes are believed to represent true specific inclusions rather than false electrostatic adducts formed during the ionization process. Due to the similarity of the behavior of these complexes in the solution and the gas phases, it may be possible to predict the $\mathrm{CD}$ selector that would produce the best separation for a select set of compounds depending on preliminary data obtained from the ESIMS. This approach would be of great advantage as finding the correct $\mathrm{CD}$ as this is a time intensive process and often requires a large quantity of expensive substituted CD's.

\section{Conclusion}

This study suggests the formation of specific host-guest inclusions between basic local anesthetics and CD's, as an extension to a former study done in this lab that suggested the formation of specific complexes between acidic barbiturates and CD's [3]. We have shown a direct correlation between the association constants of six local anesthetics with three different $\mathrm{CD}^{\prime} \mathrm{s}$ as calculated from $\mathrm{CE}$ and the complexed fractions of the CD's as calculated from the ESI-MS, which suggests that ESI-MS can be used to evaluate solution phase noncovalent complexes. The correlation indicates the possibility of predicting the migration order and the behavior of analyte-CD complexes based on the data obtained from the MS. This information would make it possible to predict the proper $\mathrm{CD}$ to use for a separation based on quick screening of various CD's using ESI-MS. It is certain that ESI-MS could be used to eliminate CD's that showed poor affinity for an analyte and would therefore, make a poor choice for further development in a CE assay.

\section{Acknowledgments}

The authors wish to thank Solvay Pharmaceuticals for their financial support of this research.

\section{References}

1. Selva, A.; Redenti, E.; Ventura, P.; Zanol, M.; Casetta, B. Study of $\beta$-Cyclodextrin-Ketoconazole-Tartaric Acid Multicomponenet Non-covalent Association by Positive and Negative 
Ionspray Mass Spectrometry. J. Mass Spectrom. 1998, 33, 729734.

2. Smith, R. D.; Light-Wahl, K. J. The Observation of NonCovalent Interactions in Solution by Electrospray Ionization Mass Spectrometry; Promise, Pitfalls, and Prognosis. Biol. Mass Spectrom. 1993, 22, 493-501.

3. Srinivasan, K.; Bartlett, M. G. Comparison of CyclodextrinBarbiturate Non-Covalent Complexes Using Electrospray Ionization Mass Spectrometry and Capillary Electrophoresis. Rapid Commun. Mass Spectrom. 2000, 14, 624-632.

4. Penn, S. G.; Goodall, D. M.; Moning, C. A.; Loran, J. S. Non-Covalent Complexes Formation of Cyclodextrins Studied by Electro Spray Ionization Mass Spectrometry. Proceedings of the 43rd ASMS Conference on Mass Spectrometry and Allied Topics; Atlanta, GA, 1995; p 236.

5. Gallagher, R. T.; Ball, C. P.; Gatehouse, D. R.; Gates, P. J.; Lobell, M.; Derrick, P. J. Cyclodextrin-Piroxicam Inclusion Complexes: Analysis by Mass Spectrometry and Molecular Modeling. Anal. Chem. 1997, 165/166, 523-531.

6. Lu, W.; Cole, R. B. Determination of Chiral Pharmaceutical Compounds, Terbutaline, Ketamine and Propranolol, by OnLine Capillary Electrophoresis-Electrospray Ionization Mass Spectrometry. J. Chromatogr. B. 1998, 714, 69-75.

7. Cunniff, J. B.; Vouros, P. False Positives in the Detection of Cyclodextrins Inclusion Complexes by Electrospray Mass-Spectrometry. J. Am. Soc. Mass Spectrom. 1995, 6, 437-447.

8. Cunniff, J. B.; Vouros, P.; Kaplan, D. L.; Fossey, S. A. The Origin and Ion of Non-covalent Peptide Dimers Observed in Liquid Secondary-Ion Mass-Spectometry Explained via FirstOrder Gas-Phase Kinetics. Biol. Mass Spectrom. 1994, 23, 741748.

9. Ramirez, J.; He, F.; Lebrilla, C. B. Gas-Phase Chiral Differentiation of Amino Acid Guests Cyclodextrin Hosts. J. Am. Chem. Soc. 1998, 120, 7387-7388.

10. Li, Y. T.; Hsieh, Y. L.; Henion, J. D.; O'Cain, T. D.; Sciehser, G. A.; Ganem, B. Analysis of the Energetics of Gas-Phase Immunophilin-Ligand Complexes by Ion Spray Mass Spectrometry. J. Am. Chem. Soc. 1994, 116, 7487-7493.

11. Ramanathan, R.; Prokai, L. Electrospray Ionization Mass Spectrometric Study of Encapsulation of Amino Acids by Cyclodextrins. J. Am. Soc. Mass Spectrom. 1995, 6, 866-871.

12. Lorenz, S. A.; Maziarz, E. P., III; Wood, T. D. Using Solution Phase Hydrogen/Deutrium (H/D) Exchange to Determine the Origin of Non-Covalent Complexes Observed by Electrospray Ionization Mass Spectrometry; in Solution or in Vacuo. J. Am. Soc. Mass Spectrom. 2001, 12, 795-804.

13. Sun, W.; Cui, M.; Liu, S.; Song, F.; Elkin, N. Electrospray Ionization Mass Spectrometry of Incubated Solutions and in
Eluates of Gel Permeation Chromatography. Rapid Commun. Mass Spectrom. 1998, 12, 2016-2022.

14. Amini, A.; Javerfalk, E.; Bastami, S.; Westerlund, D. Simultaneous Separation and Enantioresolution of Racemic Local Anesthetic Drugs by Capillary Zone Electrophoresis with Tween 20 and Methyl- $\beta$-Cyclodextrin as Selectors, Employing a Double Plug Technique. Electrophoresis 1999, 20, 204-211.

15. Valtcheva, L.; Mohammad, J.; Pettersson, G.; Hjerten, S. Chiral Separation of Beta-Blockers by High-Performance Capillary Electrophoresis Based on Non-Immobilized Cellulase as Enantioselective Protein. J. Chromatogr. 1993, 638, 263-267.

16. Tanaka, Y.; Terabe, S. Partial Separation Zone Technique for the Separation of Enantiomers by Affinity Electrokinetic Chromatography with Proteins as Chiral Pseudo-Stationary Phases. J. Chromatogr. A. 1995, 694, 277-284.

17. Amini, A.; Paulsen-Sorman, U. Enantioseparation of Local Anesthetic Drugs by Capillary Zone Electrophoresis with Cyclodextrins as Chiral Selector Using a Partial Filling Technique. Electrophoresis 1997, 18, 1019-1025.

18. Amini, A.; Wiserma, B.; Westerlund, D.; Paulsen-Sorman, U. Determination of the Enantiomeric Purity of S-Ropivacaine by Capillary Electrophoresis with Methyl- $\beta$-Cyclodextrin as Chiral Selector Using Conventional and Complete Filling Techniques. Eur. J. Pharm. Sci. 1999, 9, 17-24.

19. Amini, A.; Petterson, C.; Westerlund, D. Enantioresolution of Disopyramide by Capillary Affinity Electrokinetic Chromatography with Human $\alpha_{1}$-Acid Glycoprotein (AGP) as Chiral Selector Applying a Partial Filling Technique. Electrophoresis 1997, 18, 950-957.

20. Timothy, J.; Dann, C., III; Brown, A. P. Separation of Enantiomers Using Vancomycin in a Countercurrent Process by Suppression of Electroosmosis. Chirality 1996, 8, 77-83.

21. Kuhn, R.; Stoecklin, F.; Erni, F. Chiral Separations by HostGuest Complexation With Cyclodextrin and Crown Ether in Capillary Zone Electrophoresis. Chromatographia 1992, 33, 3236.

22. Camilleri, P. Capillary Electrophoresis Theory and Practice. CRC Press: London, 1993, p 129.

23. Chang, T. T.; Lay, J. O.; Francel, R. J. Electrokinetic Separations with Miceller Solutions and Open-Tubular Capillaries. Anal. Chem. 1984, 56, 111-113.

24. Kaneta, K.; Tanaka, S.; Taga, M.; Yoshida, H. Migration Behavior of Inorganic Anions in Micellar Electrokinetic Capillary Chromatography Using a Cationic Surfactant. Anal. Chem. 1992, 64, 798-801.

25. Haskins, N. J.; Saunders, M. R.; Camilleri, P. The Complexation and Chiral Selectivity of 2-Hydroxypropyl- $\beta$-cyclodextrin with Guest Molecules as Studied by Electrospray Mass Spectrometry. Rapid Commun. Mass Spectrom. 1994, 8, 423-426. 